\title{
Dendritic Crystallization: Numerical Study of the One-Sided Model
}

\author{
Y. Saito, ${ }^{(a)}$ G. Goldbeck-Wood, and H. Müller-Krumbhaar \\ Institut für Festkörperforschung der Kernforschungsanlage Jülich, D-5170 Jülich, West Germany
}

(Received 10 November 1986)

\begin{abstract}
Dendritic growth of crystals in two dimensions is analyzed by a Green's-function method. A one-sided diffusional model is treated in quasistationary approximation. Anisotropy is found necessary for dendritic growth. We find scaling with anisotropy to be in agreement with recent results for needle crystals in the symmetric model. For fixed anisotropy the growth rate is proportional to the square of the Peclet number. The side-branch pattern is in qualitative agreement with experiments in three dimensions for different anisotropies.
\end{abstract}

PACS numbers: $61.50 . \mathrm{Cj}, 05.70 . \mathrm{Fh}, 68.70 .+\mathrm{w}, 81.30 . \mathrm{Fb}$

The selection mechanism for dendritic patterns ${ }^{1}$ in crystal growth has been a puzzle for many years. If we ignore surface tension and crystalline anisotropy, a continuous set of parabolic shapes for the interface between a crystal growing into a supercooled melt are solutions ("Ivantsov parabolas") to the Stefan problem of a moving interface in a diffusion field.

The introduction of surface tension and crystalline anisotropy was recently found ${ }^{2-8}$ to select a needle-shaped, almost parabolic form from this continuous set of Ivantsov parabolas, while in previous approximate treatments ${ }^{1}$ of the surface tension the continuous set seemed to survive. In fact, surface tension causes an essential singularity, turning the mathematical problem into a nonlinear eigenvalue problem. The result is a discrete set of needle solutions. ${ }^{2-7}$ A recent stability analysis ${ }^{5}$ of the needle solutions indicates that the fastest-moving solution is stable in the moving frame of reference and thus is the natural candidate for the true dendrite. Experiments, ${ }^{9-11}$ on the other hand, essentially always show dendrites, i.e., needles with side branches.

The presently available results on the existence of a needle-crystal solution are usually based on the symmetric model, ${ }^{3,4,7}$ where diffusion of heat takes place symmetrically both in the solid and in the melt. Experiments in two dimensions ${ }^{9}$ are usually based on the onesided diffusion of material within the liquid rather than on diffusion of heat. A recent analysis ${ }^{6}$ of the one-sided model shows the relation between the needle solution for the crystallization and the Saffmann-Taylor problem, but is not yet as detailed as the results on the symmetrical model. The results on the boundary-layer model ${ }^{8}$ (BLM) are not conclusive in the experimentally interesting range of small deviation from equilibrium. Despite some remarkable progress, up to now it is still a completely open problem how to describe the mode of operation of a side-branch-producing dendrite, the standard situation in experiments. The best results for free dendritic growth were obtained in three dimensions ${ }^{11}$; in two dimensions there are problems with the nonplanarity of the interface normal to the side walls. ${ }^{9}$ A further prob- lem is the quantitative control of anisotropy both in two and three dimensions.

We have performed a numerical simulation of the full diffusional problem in two dimensions on the one-sided model. This is relevant for comparison with experiments on chemical diffusion. The well-known model ${ }^{1,6}$ is defined as follows:

$$
\begin{aligned}
& \partial u(x, z, t) / \partial t \equiv 0=\nabla^{2} u+(v / D) \partial u / \partial z, \\
& u_{s}=\Delta-d_{0}\left[1+\varepsilon_{m} \cos (m \theta)\right] K, \\
& -D \hat{\mathbf{n}} \cdot \nabla u_{s} \approx v \hat{n}_{z} .
\end{aligned}
$$

(1) is the diffusion equation in quasistationary approximation in a frame of reference moving at velocity $v$ in the $z$ direction. (2) is the boundary condition for the diffusion field $u$ at the interface; at infinity one has $u=0$. (3) is the conservation law for the solute or impurity at the interface. $u(x, z, t)$ is the normalized diffusion field, ' $\Delta=0-1$ is the normalized supercooling, $D$ the diffusion coefficient, $d_{0}$ the capillary length, $\varepsilon_{m}$ the strength of the $m$-fold crystalline anisotropy, and $K$ the curvature of the interface. For $d_{0}=0$ one obtains Ivantsov parabolas with tip radius $R_{0}=p l$, where $l=2 D / v$ is the diffusion length, and the Peclet number $p$ is obtained from $\Delta=\sqrt{\pi p} \exp (p) \operatorname{erfc}(\sqrt{p})$. In principle the anisotropy also enters in (3), but in the limit of small $\Delta$ (or for a segregation coefficient $\approx 1$ ) we may ignore it for better comparison with existing results on the two-sided thermal model. (1) then is defined on the liquid side of the interface only.

The use of the quasistationary approximation here is physically meaningful ${ }^{l}$ for parameter values where the diffusion length is large compared to other typical length scales like radius of the tip of the dendrite or distance between side branches. At least for the smaller supercoolings used here this is definitely the case.

We have converted (1)-(3) into a form ${ }^{12}$ such that the two-dimensional differential equation (1) becomes a one-dimensional integral equation (Green's function). The basic numerical procedure then is the following. We start with a parabola pointing in the $z$ direction symmet- 
rical about the $z$ axis, discretize it into an $N$-gon, and define $u_{s}(x, z, t=0)$ along this curve from (2). The velocity $v$ is first calculated from the corresponding Ivantsov parabola. We now know the Green's function and derivative and are left with an $N \times N$ matrix $(N<1000)$ as a linear problem for the normal velocities at each point along the interface. Solving this we define a time step $\delta t$ and displace the interface in the normal direction. We now have the new velocity from the tip of the deformed parabola and can repeat the process, simulating growth of the crystallization front.

To handle the asymptotic tail of the dendrite we have divided the interface into three parts along $z$, a tip region, a transition region, and the tail. The tail is an Ivantsov parabola of length $>5 l$ defined by the growth rate $v$. It is displaced along with the tip. Careful handling of this tail is important for the global conservation of the field variable (chemical concentration or energy), as was also noticed in Ref. 7. The missing side-branch structure there does not seem to be important as we conclude from extrapolations to infinite system size, but it may affect the noise in the system. The tip region is fully treated as described above. In the transition region at each time step the diffusion flux is calculated as above, but the displacement of each point is continuously modified down the curve such that it always connects to

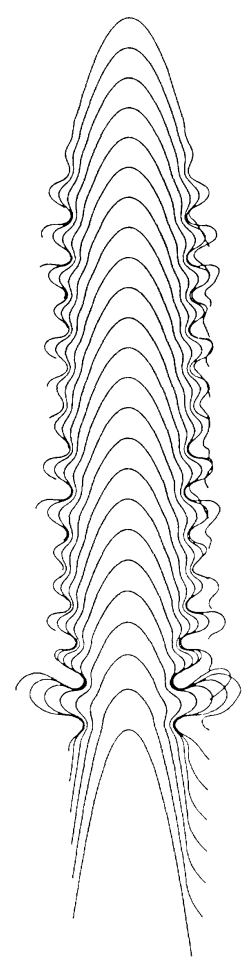

FIG. 1. Stroboscopic plot of the simulation of an almost parabolic dendrite. Parameters are $\Delta=0.25, \varepsilon_{4}=0.1$. Graphs were produced at equal time intervals. the tail. One type of test performed was to start with an Ivantsov parabola for $d_{0}=0$, calculate for a single time step the normal velocities, and compare them with the known exact values. The relative precision was better than $5 \times 10^{-4}$ at each point. Time step and grid spacing are dynamically adjusted, the typical grid spacing being $<0.05 R_{0}$, smaller than used for the needle-crystal calculation. ${ }^{7}$ The grid adjustment was already tested on previous calculations on the geometrical model. Two different, independently written versions ${ }^{12}$ of the program gave the same results for $\Delta=0.5$. Further details of the numerical procedure will be published elsewhere.

The result of such a calculation is shown in Fig. 1 as stroboscopic pictures at equal time intervals with $d_{0}=0.001$. The other parameters, $\Delta=0.25, \varepsilon_{4}=0.1$, are comparable to typical experimental values, since from $\Delta$ a Peclet number $<0.03$ follows, while $\varepsilon_{4}$ corresponds to the anisotropy of the capillary length of succinonitrile. The left-hand side in the growth direction shows the tip region only, the right-hand side includes the transition region, and the parabolic tail is not shown. The dendrite converges to a stationary side branch producing a state independent of initial conditions. The dependence of the results on the length of the tip region considered was very weak, as long as $\approx 3$ side branches were contained. We have varied the length of the tip region, the grid spacing, and the initial condition for each of the parameter sets $\Delta=(0.5,0.25), \varepsilon_{4}=(0.05,0.1,0.15)$ for an extrapolation to infinite length and zero mesh.

The results for the scaled growth rates versus anisotropy are shown in Fig. 2, compared with the needle results ${ }^{7}$ for the symmetrical model at $\Delta \longrightarrow 0$. Dendritic growth requires nonzero anisotropy. The raw data already scale with $\varepsilon_{4}$ as predicted ${ }^{7}$ apart from a constant

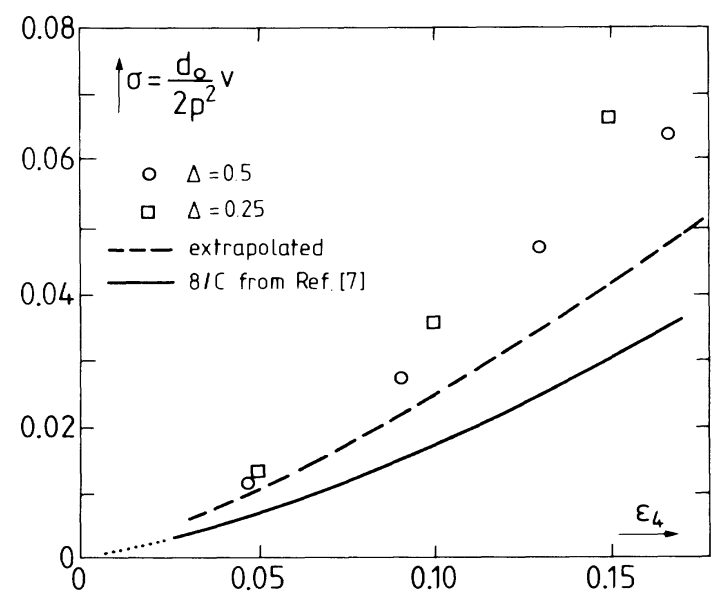

FIG. 2. Normalized growth rate vs anisotropy for $\Delta=0.25$, 0.5 . The broken line is our extrapolation to zero grid spacing and infinite size; the full curve corresponds to the needle (Ref. 7) in the symmetrical model. The scaling appears to be universal. 


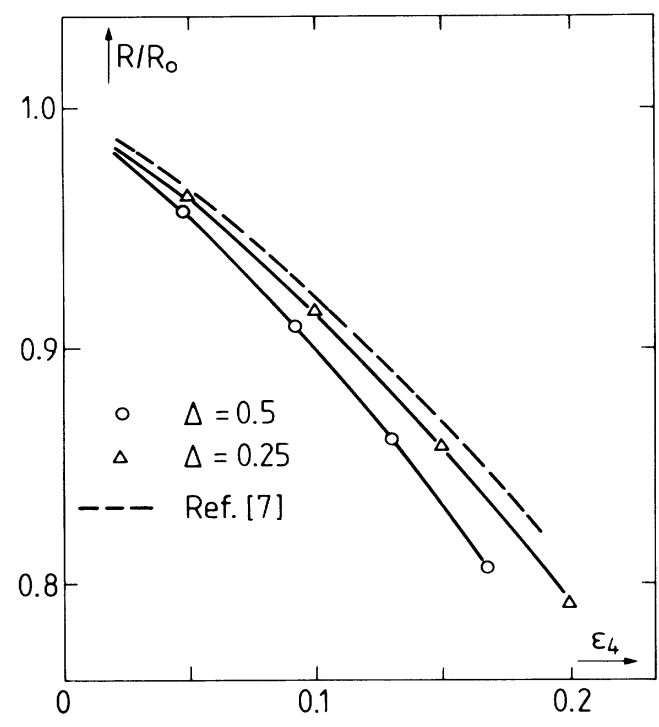

FIG. 3. Ratio of actual tip radius $R$ to Ivantsov radius $R_{0}$ corresponding to the same growth rate, plotted vs anisotropy. Again the scaling appears to be universal.

factor of $\approx 2$; the extrapolated data (broken line) are only by a constant factor of $\approx 1.3$ higher than the prediction. We estimate a maximal error of $20 \%$ for the extrapolation.

If we were to replace $p^{2} \rightarrow \Delta^{4}$ in the definition of $\sigma$ (as suggested in the limit $p \rightarrow 0$ ), the scaled numerical data obtained at two $\Delta$ values would differ by a factor 3 ! This shows that the results scale as $v \sim p^{2}$ at least up to $\Delta=0.5$, in agreement with a recent prediction for the needle. ${ }^{13}$

In Fig. 3 we show the ratio of the actual tip radius $R$ and the corresponding Ivantsov radius $R_{0}$ versus anisotropy in comparison with data for the symmetrical needle. ${ }^{7}$ Again we find striking agreement.

To summarize, we have found excellent agreement with the predictions from the symmetrical needle solution ${ }^{7}$ considering scaling of the growth rate and tip radius with the strength of the anisotropy and scaling $v \sim p^{2}$ with the Peclet number. Furthermore we confirm scaling of the spacing between side branches $\approx 3 R_{0}$ at $\varepsilon_{4} \approx 0.1$ in agreement with experiments in three dimensions. ${ }^{11}$ The amplitude of the side branches ${ }^{12}$ decreases with increasing anisotropy, as also observed in experiments on different material. ${ }^{11}$ These results are insofar remarkable, as the pattern Fig. 1 with the side branches is very different from the needle solutions. This quantitative agreement indicates that the selection process here is largely governed by the immediate neighborhood of the tip. At the moment we cannot answer the question $^{5,14}$ whether the side branches are triggered by noise.
Noise in our calculation comes from two sources, the discretization and the irregularities due to competing side branches. A refined version of the program starting from a precise needle crystal might elucidate this in the near future. Considering experimental uncertainties in materials constants, hydrodynamic flow, and boundary conditions, we finally think that our calculations are at least competitive.

We thank Martine Ben-Amar for providing her data in Fig. 3.

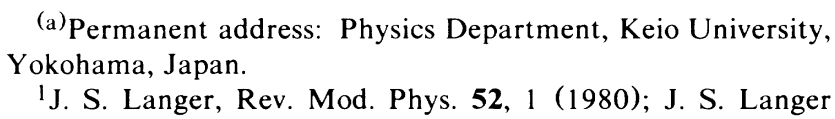
${ }^{1}$ J. S. Langer, Rev. Mod. Phys. 52, 1 (1980); J. S. Langer
nd H. Müller-Krumbhaar, Acta. Metall. 26, 1681, 1689, 1697 (1978); H. Müller-Krumbhaar and J. S. Langer, Acta. Metall. 29, 145 (1981).

${ }^{2}$ P. Pelce and Y. Pomeau, to be published.

${ }^{3}$ D. C. Hong and J. S. Langer, Phys. Rev. Lett. 56, 2032 (1986).

${ }^{4}$ D. Kessler, J. Koplik, and H. Levine, Proceedings of the NATO Advanced Research Workshop on Pattern, Defects and Microstructures in Non-Equilibrium Systems Austin, Texas, March 1986 (unpublished); D. Kessler and H. Levine, Phys. Rev. A 33, 2621, 2634 (1986).

${ }^{5}$ D. Kessler and H. Levine, Phys. Rev. Lett. 57, 3069 (1986).

${ }^{6}$ D. Kessler, J. Koplik, and H. Levine, Phys. Rev. A 34, 4980 (1986).

${ }^{7}$ M. Ben-Amar and Y. Pomeau, Europhys. Lett. 2, 307 (1986), and "Theory of the Needle Crystal" (to be published).

${ }^{8}$ E. Ben-Jacob, N. Goldenfeld, J. S. Langer, and G. Schön, Phys. Rev. Lett. 51, 1930 (1983), and Phys. Rev. A 29, 330 (1984); E. Ben-Jacob, N. Goldenfeld, B. G. Kotliar, and J. S. Langer, Phys. Rev. Lett. 53, 2110 (1984).

${ }^{9}$ H. Honjo, S. Ohta, and Y. Sawada, Phys. Rev. Lett. 55, 841 (1985).

${ }^{10}$ E. Ben-Jacob, R. Godbey, N. Goldenfeld, J. Koplik, H. Levine, T. Mueller, and L. Sander, Phys. Rev. Lett. 55, 1315 (1985).

${ }^{11}$ M. E. Glicksman, R. Schaefer, and J. Ayers, Metall. Trans. A 7, 1747 (1976); S. Huang and M. Glicksman, Acta. Metall. 29, 701, 717 (1981); M. E. Glicksman and B. Singh, in Solidification and Fluid-Dynamics Proceedings of a workshop at Giesserei-Institut der Rheinisch-Westfälische Technische Hochschule Aachen, edited by P. Sahm and Y. Malmejac (Giesserei-Institut, Aachen, 1984), p. 182.

${ }^{12} \mathrm{G}$. Goldbeck, "Strukturbildung bei dendritischer Kristallisation" (unpublished); H. Müller-Krumbhaar, G. Goldbeck, and Y. Saito, in "Proceedings of the OJI Seminar on Crystal Morphology and Growth Units, Yamagata, Japan, 1985," edited by I. Sunagawa (unpublished).

${ }^{13}$ B. Caroli, C. Caroli, C. Misbah, and B. Roulet, to be published.

${ }^{14}$ R. Pieters and J. S. Langer, Phys. Rev. Lett. 56, 1948 (1986). 